\title{
Absorption efficiency of gold nanorods determined by quantum dot fluorescence thermometry
}

\author{
L. M. Maestro, ${ }^{1}$ P. Haro-González, ${ }^{2}$ J. G. Coello, ${ }^{3}$ and D. Jaque ${ }^{1}$ \\ ${ }^{1}$ Fluorescence Imaging Group, Departamento de Física de Materiales, Instituto Nicolás Cabrera, \\ Facultad de Ciencias, Universidad Autónoma de Madrid, 28049 Spain \\ ${ }^{2}$ Departamento de Física Fundamental y Experimental, Electrónica y Sistemas, Universidad de La Laguna, \\ Tenerife, Spain \\ ${ }^{3}$ Departament of Physics and Astronomy, University College London, London, United Kingdom
}

(Received 16 March 2012; accepted 30 April 2012; published online 17 May 2012)

\begin{abstract}
In this work quantum dot fluorescence thermometry, in combination with double-beam confocal microscopy, has been applied to determine the thermal loading of gold nanorods when subjected to an optical excitation at the longitudinal surface plasmon resonance. The absorbing/heating efficiency of low $(\approx 3)$ aspect ratio gold nanorods has been experimentally determined to be close to $100 \%$, in excellent agreement with theoretical simulations of the extinction, absorption, and scattering spectra based on the discrete dipole approximation. (C) 2012 American Institute of Physics. [http://dx.doi.org/10.1063/1.4718605]
\end{abstract}

Gold nanorods (GNRs) are one of the most popular metallic nanoparticles with numerous applications in a great variety of fields including data storage, solar energy, catalysis, photonic devices, and microscopy. ${ }^{1-3}$ In particular, they are especially useful in biomedical applications due to their multi-functional character. ${ }^{4,5}$ This is related to the different physical phenomena that are activated after optical excitation of their surface plasmon resonance (SPR). When electromagnetic radiation of wavelength equal to the SPR wavelength $\left(\lambda_{\mathrm{SPR}}\right)$ illuminates a GNR, it promotes the collective and synchronized motion of its surface charges. When this process is activated by high power laser pulses, the currentinduced surface field enhancement could be strong enough to promote the population of the high-energy bulk levels of gold, leading to the appearance of a visible luminescence. This visible luminescence that follows a nonlinear relation in respect to the excitation power has been widely used in the past for high contrast multiphoton-excited imaging of living cells under optical excitation within the so-called "biological window" $(700-900 \mathrm{~nm}) .{ }^{6}$ In addition to their application in bio-imaging, GNRs have been also demonstrated to be efficient photo-thermal agents. ${ }^{7}$ In this case, the heat delivered during the relaxation of surface currents induces local heating of the biological system in which the GNR are incorporated. This plasmon-assisted laser-induced local heating has been already used for thermal therapy of cancel cells and tumors by using low laser powers. ${ }^{8}$ Thermal therapies require a full control over the local heating produced in the bio-system so that it would be driven just to the cytotoxic thermal level $\left(40-45^{\circ} \mathrm{C}\right)$. Larger increments would lead to the appearance of undesirable collateral damage in adjacent cells and tissues. ${ }^{9}$ In GNR-assisted thermal therapies, temperature increment is given by some parameters that can be controlled via experiment design (such as the number of GNRs incorporated in the bio-system and the laser power) but also by the intrinsic properties of the GNRs. ${ }^{10}$ In particular, the resulting laser-induced thermal loading depends on the heating efficiency of GNRs (the fraction of the laser power reaching the GNR that is converted into heat). Due to the very low fluorescence efficiency of GNRs, almost all optical power absorbed by the GNR is converted into heat. Therefore, the heating efficiency of GNRs is given by the absorption efficiency of GNRs, $\Phi_{\text {abs }}$, here defined as the relative contributions of absorption coefficient $\left(\alpha_{\mathrm{abs}}\right)$ to the net extinction coefficient of GNRs $\left(\alpha_{\mathrm{ext}}=\alpha_{\mathrm{abs}}+\alpha_{\mathrm{sct}}\right.$, being $\alpha_{\mathrm{scat}}$ the scattering coefficient), i.e., $\Phi_{\text {abs }}=\alpha_{\mathrm{abs}} / \alpha_{\mathrm{ext}}$. Theoretical models have stated that this magnitude is strongly dependent on the particular size and shape of GNRs ${ }^{10-12}$ so that an adequate chose of GNR dimensions is required for the optimization of GNR based photo-thermal treatments. Nevertheless, despite its interest from the applied and fundamental points of view, experimental works to determine $\Phi_{\text {abs }}$ for GNRs are very limited.

In this work, quantum dot fluorescence (non-contact) thermometry in combination with double-beam confocal microscopy has been applied to determine the laser induced thermal loading of GNRs aqueous solutions in the presence of SPR optical excitation. Comparison between experimental data and theoretical models, concerning thermal loading effects in microfluidics, allowed us to determine the heating efficiency of GNRs. The absorption, scattering, and extinction spectra have been simulated based on the discrete dipole approximation (DDA), and from them the absorption efficiency calculated and compared to that obtained experimentally. ${ }^{12,13}$

The GNRs under investigation in this work were provided by NanoPartz Inc. The GNRs were dispersed into distilled water at a concentration $1.7 \times 10^{12} \mathrm{~cm}^{-3}$. The extinction spectrum of the GNR solution, after subtraction of the water absorption background, is shown in Fig. 1(a). The size dispersion histograms (as obtained from the analysis of TEM images) are included in Figs. 1(b) and 1(c). The GNRs used in this work have a mean radius and length of 12 and $47 \mathrm{~nm}$, respectively. In both dimensions the dispersion was found to be close to $\pm 15 \%$. A characteristic GNR is shown in detail in the inset of Fig. 1(a). In the extinction coefficient of Fig. 1(a) two extinction peaks, corresponding to the longitudinal and transversal SPR modes, are observed. The extinction peak corresponding to the transversal SPR is 


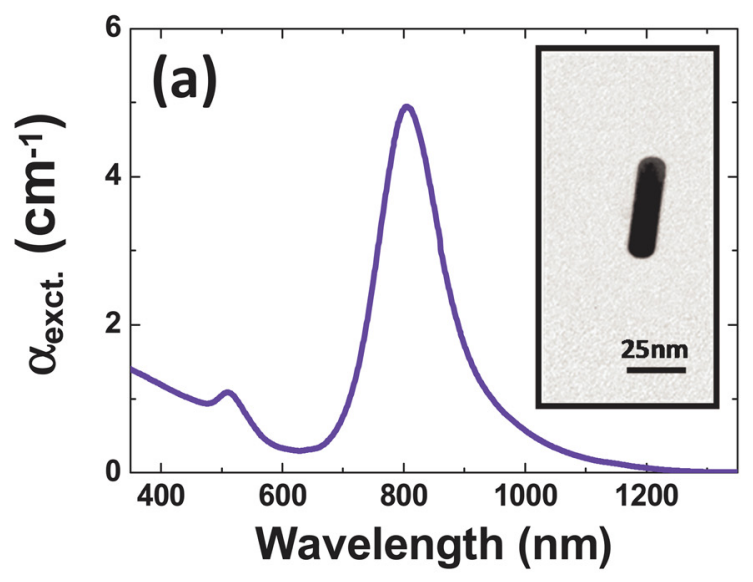

located at $500 \mathrm{~nm}$ whereas the longitudinal mode resonance appears at $808 \mathrm{~nm}\left(\lambda_{S P R}=808 \mathrm{~nm}\right)$. From data included in Fig. 1 the extinction coefficient at $\lambda_{S P R}$ was found to be $4.9 \mathrm{~cm}^{-1}$. For the purpose of fluorescence thermal sensing experiments, CdSe quantum dots $(4 \mathrm{~nm}$ in diameter and provided by Invitrogen Inc.) were added to the solutions resulting in a final CdSe-QD concentration as low as $4 \times 10^{9} \mathrm{~cm}^{-3}$. This concentration was so small that the presence of CdSe-QDs does not modify the extinction spectrum from that shown in Fig. 1(a). The solution containing GNRs and CdSe-QDs showed a very stable colloidal behavior without any evidence of precipitation during months. The emission spectrum of the GNRs+QDs solution after $488 \mathrm{~nm}$ excitation consists of a broad band centered at around $640 \mathrm{~nm}$ whose peak wavelength shifts linearly with temperature at a rate of $0.1 \pm 0.05 \mathrm{~nm} /{ }^{\circ} \mathrm{C}$ (see Fig. 2(a)). ${ }^{14}$ The mixed solution was placed within a microscope slide containing a $200 \mu \mathrm{m}$ height and $2 \mathrm{~mm}$ width $\mu$-channel (provided by Ibidi Inc.). The microscope slide was placed in a doublebeam microscope as it is schematically shown in Fig. 2(b). The solution was locally excited by a single-mode fibercoupled continuous wave laser operating at $808 \pm 1 \mathrm{~nm}$. Infrared radiation was focused within the $\mu$-channel with a $4 \times, 0.1$ NA microscope objective, leading to a spot size of $5 \mu \mathrm{m}$. In order to determine the laser-induced temperature increment produced at focus, a $488 \mathrm{~nm}$ continuous wave laser was also focused into the solution by using a second microscope objective $(10 \times, 0.25 \mathrm{NA})$ leading to a spot size close to $1.2 \mu \mathrm{m}$, spatially overlapping with the infrared (heating) spot. The CdSe-QD fluorescence, generated after the $488 \mathrm{~nm}$ excitation, was collected by the $10 \times$ microscope objective, and after passing through different filters, it was spectrally analyzed by a high resolution spectrometer. The axial and lateral resolutions in the fluorescence detection system have been calculated to be 12.5 and $1.2 \mu \mathrm{m}$, respectively. The spectral resolution has been estimated to be $0.1 \mathrm{~nm}$ so that we were able to determine the local temperature of the GNR+QD solution (from the analysis its CdSe-QD fluorescence) with a resolution of $1{ }^{\circ} \mathrm{C}$. Fig. 2(b) also includes a typical micro-fluorescence CdSe-QD spectrum obtained in our experimental set-up when the infrared (heating) laser was switched on and off, i.e., in the absence/presence of optical excitation of the SPR. It is clear that the excitation of the SPR causes a relevant on-focus temperature increment that shifts the CdSe-QDs toward longer wavelengths.
Previous models concerning laser-induced thermal loading of microfuidics concluded that the laser-induced temperature increment at focus, $\Delta T_{\text {focus }}$, can be evaluated as a function of the laser input power, $P_{i n}$, by ${ }^{15}$

$$
\Delta T_{\text {focus }}=\frac{P_{\text {in }} \cdot \alpha_{a b s}}{2 \cdot \pi \cdot K} \cdot \operatorname{Ln}\left[\frac{D}{w_{l}}\right]=\frac{P_{\text {in }} \cdot \Phi_{a b s} \cdot \alpha_{\text {ext }}}{2 \cdot \pi \cdot K} \cdot \operatorname{Ln}\left[\frac{D}{w_{l}}\right],
$$

where $\alpha_{a b s}=\Phi_{a b s} \cdot \alpha_{e x t}$ is the absorption coefficient at laser wavelength, $\alpha_{e x t}$ is the total extinction coefficient at laser
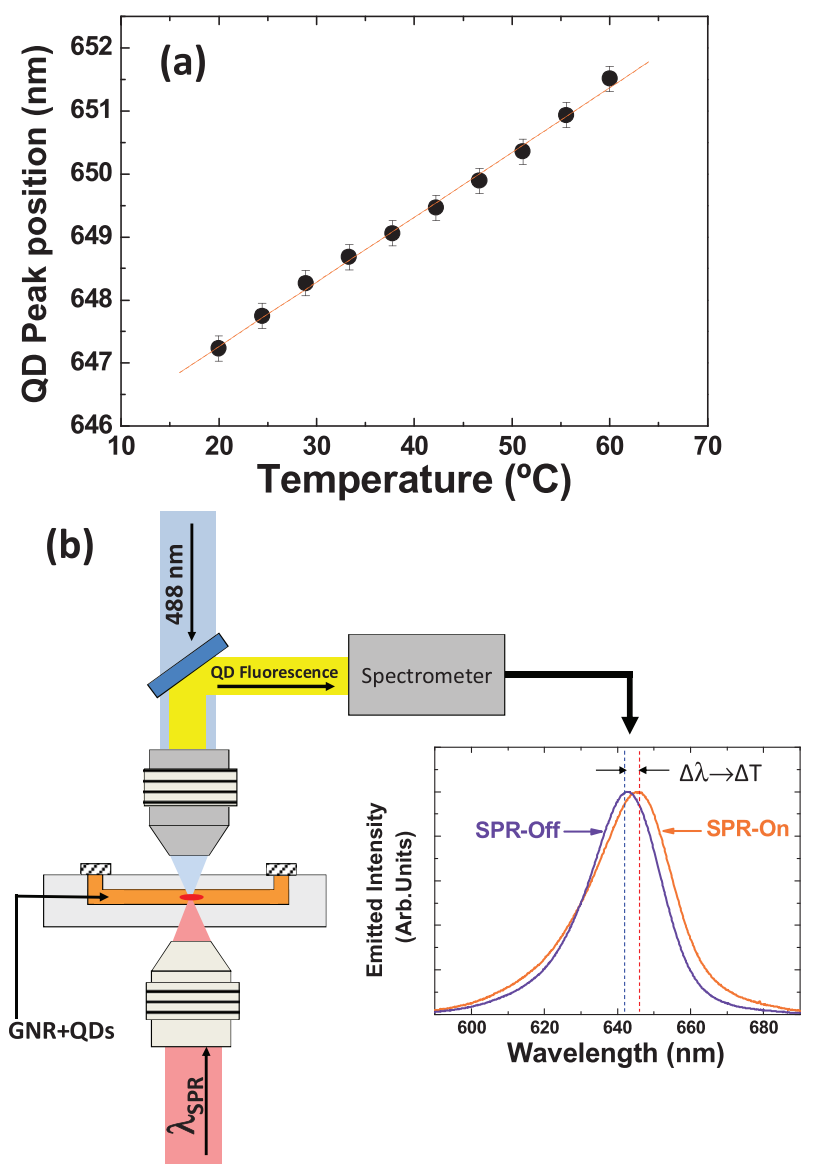

FIG. 2. (a) Peak emission wavelength of the CdSe-QD fluorescence as a function of the GNR solution temperature. Dots are experimental data and dashed line is the best linear fit. (b) Left: schematic diagram of the doublebeam confocal microscope used in this work. Right: fluorescence emission spectrum obtained for the CdSe-QDs incorporated into the GNR solution under $488 \mathrm{~nm}$ excitation in the presence/absence of optical excitation of the surface plasmon resonance of GNRs. 
wavelength, $K$ is the thermal conductivity of water $\left(0.6 \mathrm{~W} \cdot \mathrm{m}^{-1} \cdot \mathrm{K}^{-1}\right), D$ is the $\mu$-channel height $(200 \mu \mathrm{m})$, and $w_{l}$ is the laser beam waist $(5 \mu \mathrm{m})$. Therefore, from the experimentally determined slope of the " $\Delta T_{\text {focus }}$ vs $P_{\text {in }}$ " data $\left(\frac{d\left(\Delta T_{\text {focus }}\right)}{d P_{\text {in }}}\right)$, it is possible to determine the absorption efficiency $\Phi_{a b s}$ of GNRs $\Phi_{a b s}=\frac{2 \cdot \pi \cdot K}{\alpha_{e x t} \cdot L n\left[D / w_{l}\right]} \cdot \frac{d\left(\Delta T_{\text {focus }}\right)}{d P_{\text {in }}}$.

Figure 3(a) shows the on-focus temperature increment produced in the GNR solution as a function of the $808 \mathrm{~nm}$ laser power. A linear relation between temperature increment and laser power is observed (see solid line), in agreement with expression (1). From the linear fit of experimental data we obtained $\frac{d\left(\Delta T_{\text {focus }}\right)}{d P_{\text {in }}}=480 \pm 10^{\circ} \mathrm{C} / \mathrm{W}$ that leads to $\Phi_{a b s}=1 \pm 0.02$. In order to verify the consistence of our approach, we repeated the same procedure with different GNR concentrations. Results are shown in the inset of Fig. 3(a). As it was expected, the absorption efficiency was found to be independent on the GNR concentration (note that $\Phi_{a b s}$ is only expected to be dependent on the GNR geometry). From data included in the inset of Fig. 3(a) the averaged absorption efficiency was calculated to be $\Phi_{a b s}=0.98 \pm 0.02$. This close-to-unit absorbing/heating efficiency is, indeed, an outstanding property of these particular GNRs for the purpose of laser-assisted thermal treatments. In
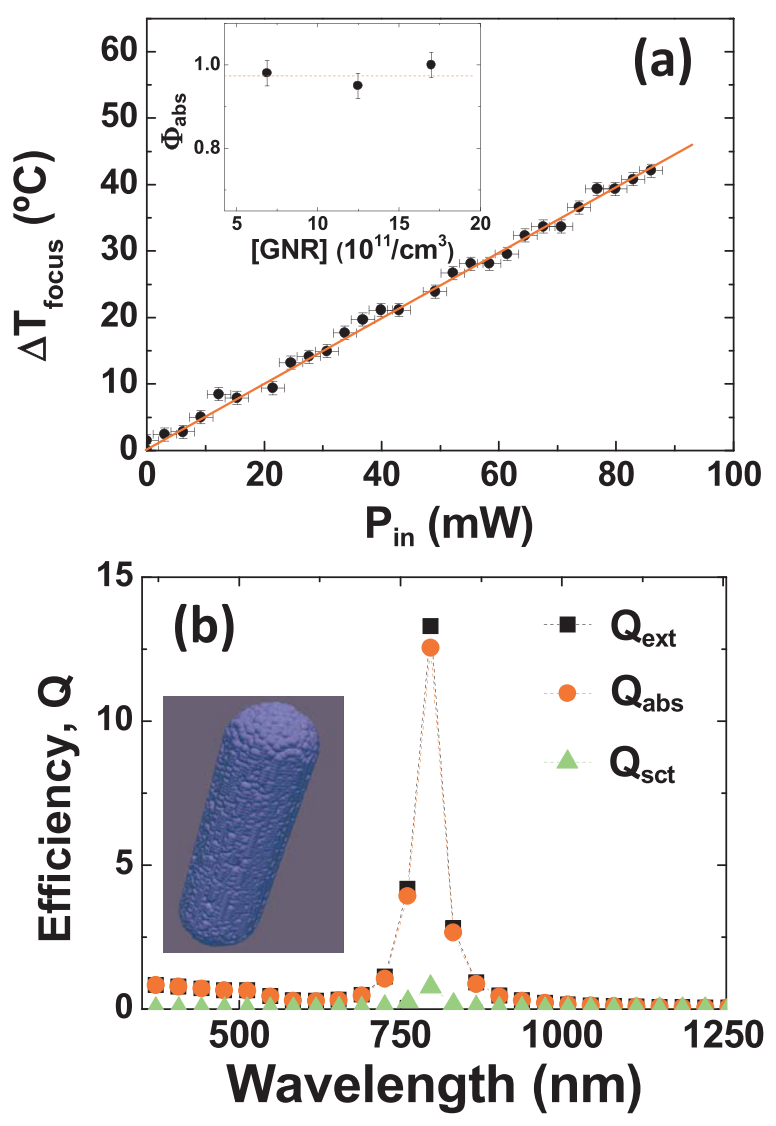

FIG. 3. (a) On focus temperature increment as a function of the $808 \mathrm{~nm}$ excitation laser power. Dots are experimental data; solid line is the best linear fit of experimental data. Inset shows the absorption efficiency $\Phi_{a b s}$ as a function of the GNR concentration. (b) Simulated absorption, scattering, and extinction efficiencies of $12 \mathrm{~nm}$ width and $47 \mathrm{~nm}$ length GNRs. Inset shows the geometrical reproduction of the GNR based on a cubic array of 38500 dipoles. addition, the SPR is excited within the biological window (700-900 nm) ensuring large penetration depths. The combination of these two features makes these GNRs ( $12 \mathrm{~nm}$ diameter and $47 \mathrm{~nm}$ long) especially suitable for efficient in-depth thermal treatments.

In order to verify the validity of our experimental approach for the $\Phi_{a b s}$ determination, we have modeled the absorption and scattering processes caused by the GNRs shown in Fig. 1. For this purpose we have adopted the DDA. ${ }^{16}$ The DDA method is a numerical method in which the object interacting with light (in our case GNRs $12 \mathrm{~nm}$ in diameter and $47 \mathrm{~nm}$ in length) is geometrically reproduced by a cubic lattice of $\mathrm{N}$ polarizable point dipoles (see inset in Fig. 3(b)). ${ }^{11,12}$ The number of point dipoles should be large enough to reconstruct a continuous surface for the incident electromagnetic radiation ( $\mathrm{N}=38500$, in our simulations). By using the code developed by Draine et al., ${ }^{17}$ we have simulated the absorption, scattering, and extinction efficiencies $\left(\mathrm{Q}_{\mathrm{abs}}, \mathrm{Q}_{\mathrm{sct}}\right.$, and $\mathrm{Q}_{\mathrm{ext}}$, respectively) of the reconstructed GNR. These are defined as the absorption, scattering, or extinction cross sections divided by the GNR effective area. The simulation results are shown in Fig. 3(b). At the SPR wavelength, DDA simulations have concluded that $\mathrm{Q}_{\mathrm{abs}}=0.91 \cdot \mathrm{Q}_{\mathrm{ext}}$. Thus, theoretical calculations lead to $\Phi_{a b s}=0.91$, in excellent agreement with the value obtained from the analysis of experimental data.

In summary, we have introduced an original approach for the experimental determination of the absorption efficiency of gold nanorods. This approach is based on the use of CdSeQDs as fluorescent nanothermometers for the determination of the thermal loading caused in a GNR solution by a tightly focused laser tuned to the surface plasmon resonance wavelength. Based on this technique, the absorption efficiency of $12 \mathrm{~nm}$ width, $47 \mathrm{~nm}$ length GNRs has been experimentally determined to be $\Phi_{a b s}=0.98 \pm 0.02$. Theoretical calculations based on the discrete dipole approximation have concluded that this experimental value is, indeed, very close to that theoretically predicted, supporting the validity of the experimental approach here presented. The particular GNRs investigated in this work emerge as promising agents for efficient large penetration laser assisted thermal treatments.

This work was supported by the Universidad Autónoma de Madrid and Comunidad Autónoma de Madrid (Project No. S2009/MAT-1756), by the Spanish Ministerio de Educacion y Ciencia (MAT2010-16161 and MAT2010-21270C04-02), FPI grant by Agencia Canaria de Investigación del Gobierno de Canarias, and by the Caja Madrid Foundation. J.G.C. was supported by the UK Engineering and Physical Sciences Research Council (EPSRC) sponsored Quantum Information Processing Interdisciplinary Research Centre (QIPIRC). Work also supported by the EU project COST Action CM1006.

\footnotetext{
${ }^{1}$ M. C. Daniel and D. Astruc, Chem. Rev. 104, 293 (2004).

${ }^{2}$ X. Li, J. Qian, L. Jiang, and S. He, Appl. Phys. Lett. 94, 063111 (2009).

${ }^{3}$ J. Chen, M. Yang, Q. Zhang, E. C. Cho, C. M. Cobley, C. Kim, C. Glaus,

L. V. Wang, M. J. Welch, and Y. Xia, Adv. Funct. Mater. 20, 3684 (2010).

${ }^{4} \mathrm{M}$. A. Garcia, in Recent Developments in Bio-Nanocomposites for Biomedical Applications, edited by A. Tiwari (NOVA, Tsukuba, Japan, 2010).
} 
${ }^{5}$ K. Kim, S.-W. Huang, S. Ashkenazi, M. O'Donnell, A. Agarwal, N. A. Kotov, M. F. Denny, and M. J. Kaplan, Appl. Phys. Lett. 90, 223901 (2007). ${ }^{6}$ A. M. Smith, M. C. Mancini, and S. M. Nie, Nat. Nanotechnol. 4, 710 (2009). ${ }^{7}$ T. B. Huff, L. Tong, Y. Zhao, M. N. Hansen, J. X. Cheng, and A. Wei, Nanomedicine 2, 125 (2007).

${ }^{8}$ H. Kang, B. H. Jia, J. L. Li, D. Morrish, and M. Gu, Appl. Phys. Lett. 96, 063702 (2010).

${ }^{9}$ G. S. Terentyuk, G. N. Maslyakova, L. V. Suleymanova, N. G. Khlebtsov, B. N. Khlebtsov, G. G. Akchurin, I. L. Maksimova, and V. V. Tuchin, J. Biomed. Opt. 14, 021016 (2009).

${ }^{10}$ G. Baffou, R. Quidant, and C. Girard, Appl. Phys. Lett. 94, 153109 (2009).
${ }^{11}$ K.-S. Lee and M. A. El-Sayed, J. Phys. Chem. B 109, 20331 (2005).

${ }^{12}$ P. K. Jain, K. S. Lee, I. H. El-Sayed, and M. A. El-Sayed, J. Phys. Chem. B 110, 7238 (2006).

${ }^{13}$ O. L. Muskens, G. Bachelier, N. Del Fatti, F. Vallee, A. Brioude, X. Jiang, and M.-P. Pileni, J. Phys. Chem. C 112, 8917 (2008).

${ }^{14}$ L. M. Maestro, E. M. Rodriguez, F. S. Rodriguez, M. C. I. la Cruz, A. Juarranz, R. Naccache, F. Vetrone, D. Jaque, J. A. Capobianco, and J. G. Sole, Nano Lett. 10, 5109 (2010).

${ }^{15}$ H. B. Mao, J. R. Arias-Gonzalez, S. B. Smith, I. Tinoco, and C. Bustamante, Biophys. J. 89, 1308 (2005).

${ }^{16}$ E. M. Purcell and C. R. Pennypac, Astrophys. J. 186, 705 (1973).

${ }^{17}$ P. J. F. Bruce T. Draine, e-print arXiv:1202.3424v2. 
Applied Physics Letters is copyrighted by the American Institute of Physics (AIP). Redistribution of journal material is subject to the AIP online journal license and/or AIP copyright. For more information, see http://ojps.aip.org/aplo/aplcr.jsp 